\title{
Spin Hall effect tunnelling spectroscopy
}

\author{
Luqiao Liu^, Ching-Tzu Chen and J. Z. Sun
}

\begin{abstract}
The spin Hall effect (SHE) and its inverse have been widely used to generate and detect spin currents ${ }^{1-8}$. To date, most experiments focus only on characterizing electrons near the Fermi surface ${ }^{4-8}$, whereas the SHE, which originates from the spin-orbit interaction, is expected to be energy dependent ${ }^{9,10}$. Here, we report a tunnelling spectroscopy technique developed to measure the SHE under finite bias voltages. We studied the SHE for typical $5 d$ transition metals. At zero d.c. bias, the obtained spin Hall angles confirm the results from spin-torque experiments ${ }^{8,11-13}$. At high bias, the transverse spin Hall signals of these materials exhibit very different voltage dependences. The SHE tunnelling spectra have important implications in pinpointing the mechanisms of the SHE and provide guidelines for engineering high-SHE materials. Moreover, SHE tunnelling spectroscopy can be directly applied to two-dimensional surface states with strong spin-orbit coupling, such as Dirac electrons in topological insulators.
\end{abstract}

Tunnelling spectroscopy is one of the most widely used experimental techniques to study electronic structures ${ }^{14}$. Conventional tunnelling spectroscopy measures the longitudinal tunnelling current to extract material parameters such as the electronic density of states (DOS) and band gap. In this Letter we show that by measuring the transverse current or voltage generated from spin-polarized tunnelling carriers, one can probe transport properties related to the spin-orbit interaction, in particular the spin Hall effect. Furthermore, the SHE signal from hot electrons can be detected by applying finite bias voltages.

To inject spin polarized current into the normal metal (NM), we pattern the NM/oxide/ferromagnet (FM) stacks into the shape shown in Fig. 1a. We apply the charge current between leads 1 and 3 and measure the voltage between 2 and 4 . In the presence of the inverse SHE (ISHE), the spin angular momentum $\sigma$, injected spin current density $j_{s}$ and generated charge current density $j_{c}$ satisfy the relationship ${ }^{3} \mathbf{j}_{\mathrm{c}} \sim \boldsymbol{\sigma} \times \mathbf{j}_{\mathrm{s}}$. Therefore, a transverse voltage is expected to arise in the $y$ direction when spins aligned in-plane along the $x$ direction are injected into the NM and diffuse along the $z$ axis (Fig. 1b). At zero d.c. bias, the ISHE voltage of the device in Fig. 1a can be written as ${ }^{15,16} V_{24}=\rho \theta_{\mathrm{SH}} j_{\mathrm{s}} a \cdot(b / w) \cdot\left(\lambda_{\mathrm{sf}} / t\right) \tanh \left(t / 2 \lambda_{\mathrm{sf}}\right)$ (see also the Supplementary Information). Here $\rho$ is the resistivity of the spin Hall ( $\mathrm{SH}$ ) metal, $\theta_{\mathrm{SH}}$ is the $\mathrm{SH}$ angle, defined as the ratio between the orthogonally related charge current density and the spin current density, and $j_{\mathrm{s}}=P j_{\text {in }}$ represents the spin current density, where $P$ and $j_{\text {in }}$ denote the spin polarization and injected chargecurrent density. $a, b, w$ and $t$ are dimensions defined in Fig. 1a. The shunting factors, $b / w$ and $\left(\lambda_{\mathrm{sf}} / t\right) \tanh \left(t / 2 \lambda_{\mathrm{sf}}\right)$, take into account that the ISHE develops only in the region under the tunnel barrier and decays over a thickness of $\lambda_{\mathrm{sf}}$ whereas the charge current flows across the entire channel cross-section of width $w$ and thickness $t$.
As $j_{\text {in }}=I_{13} /(a b)$, we arrive at the following expression for the transverse resistance:

$$
\frac{\mathrm{d} V_{24}}{\mathrm{~d} I_{13}}=\frac{\theta_{\mathrm{SH}} P \rho}{w} \cdot \frac{\lambda_{\mathrm{sf}}}{t} \cdot \tanh \left(t / 2 \lambda_{\mathrm{sf}}\right)
$$

Figure 1d shows the circuit for detecting the transverse resistance (Methods). Because of imperfect alignment in the photolithography, there exists a parasitic voltage on top of the ISHE signal, which we compensate with a resistance bridge. Because of its geometric nature, the parasitic resistance remains constant with magnetic field, and the $\mathrm{SH}$ resistance has been observed to be independent of its sign or magnitude in all devices.

Figure $2 \mathrm{a}$ shows the ISH resistance $(\mathrm{d} V / \mathrm{d} I)_{13,24}$ curve of a $\mathrm{Ta} / \mathrm{MgO} / \mathrm{CoFeB}$ sample. A hysteretic loop develops when we sweep the magnetic field along the $x$ direction $\left(0^{\circ}\right)$, and vanishes when we sweep along the $y$ direction $\left(90^{\circ}\right)$, consistent with the symmetry of the ISHE. The coercivity of $\sim 20$ Oe agrees with the switching field of the CoFeB layer (Supplementary Information). Figure 2b shows the transverse resistance $(\mathrm{d} V / \mathrm{d} I)_{24,13}$ measured in the direct SHE (DSHE) configuration, in which we apply the current between leads 2 and 4 and measure the voltage between leads 1 and 3. We can see that the magnitude of the signals agrees well between the DSHE and ISHE configurations, as is expected from the Onsager reciprocal relation. In the DSHE, the voltage across the barrier is induced by spin accumulation at the Ta surface (see inset of Fig. 2b). Using the model of ref. 15, we can write the DSHE-induced voltage as $V_{13}=\left(\theta_{\mathrm{SH}} P \rho \lambda_{\text {sf }} / w t\right) \cdot I_{24} \tanh \left(t / 2 \lambda_{\text {sf }}\right)$, the same as the ISHE equation (Supplementary Information).

Figure 3a gives the ISHE data of a device with an $\mathrm{AlO}_{x}$ tunnel barrier $\left(\mathrm{Ta} / \mathrm{AlO}_{x} / \mathrm{CoFeB}\right)$. At zero bias (black circles in Fig. 3a), the magnitude of the signal is about half that of the $\mathrm{MgO}$ device $(1.3 \mathrm{~m} \Omega$ versus $2.6 \mathrm{~m} \Omega$ ). This can be explained by the different values of spin polarization across those two barriers. From the tunnelling magnetoresistance (TMR) of standard magnetic tunnel junctions (MTJs) made of these two barriers, we determined $P(\mathrm{MgO}) \sim 0.60 \pm 0.05$ and $P\left(\mathrm{AlO}_{x}\right) \sim 0.38 \pm 0.05$ (Supplementary Information), roughly consistent with the difference in the ISH resistances.

Besides Ta, we also measured samples made from Pt. Previous studies showed that both metals have a strong SHE but their signs are opposite ${ }^{8,10,16}$. As shown in Fig. 4a (ISHE) and 4b (DSHE), the transverse resistances of the $\mathrm{Pt} / \mathrm{MgO} / \mathrm{CoFeB}$ sample indeed exhibit opposite signs to those of the Ta samples. This fact also indicates that the measured signal must originate from the NM layer rather than from magneto-transport effects in the CoFeB layer, which would have resulted in the same sign in transverse resistance (Supplementary Information). To extract the SH angles, we note that all the parameters in equation (1) can be determined relatively precisely in our experiment except for $\lambda_{\text {sf }}$. Large discrepancies exist in the values of $\lambda_{\text {sf }}$ reported from different experiments. To 
a

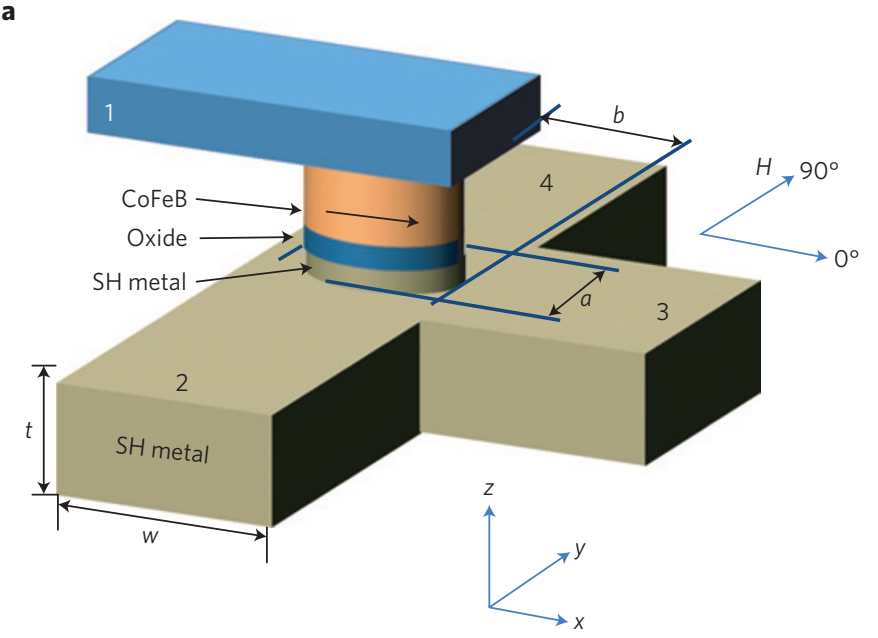

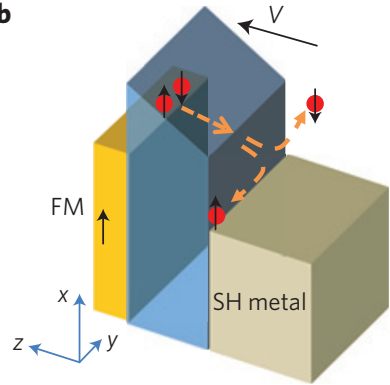

C

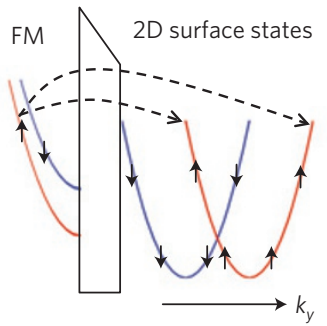

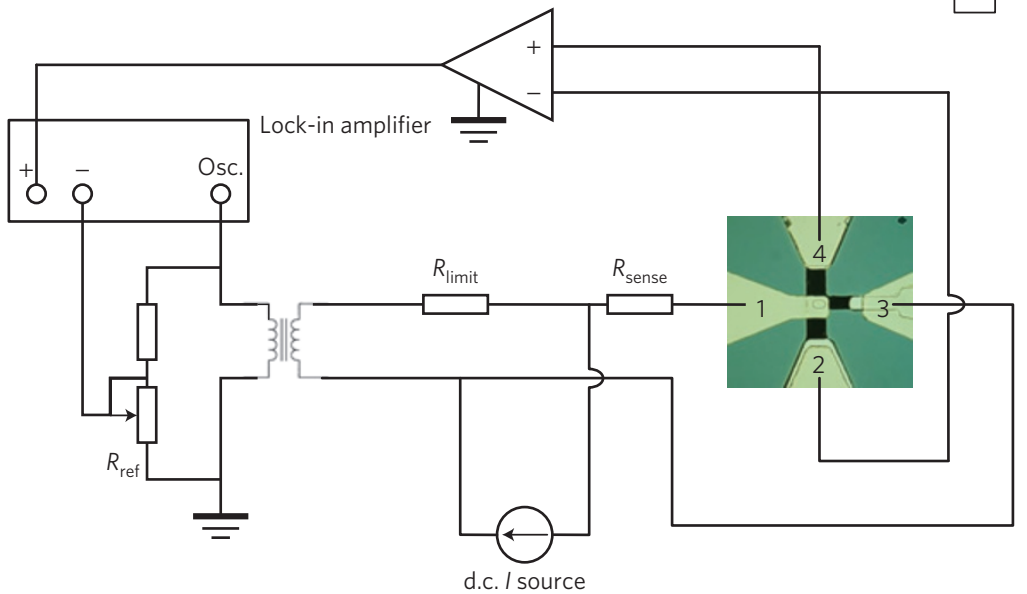

Figure 1 | Overview of the device and the circuit for SHE tunnelling spectroscopy measurements. a, Schematic of the devices used in the SHE tunnelling spectroscopy experiment. The lateral dimensions of the tunnel junction are defined by $a$ and $b . w$ and $t$ represent the width and thickness of the SH metal channel. b,c, Illustration of the generation of transverse current for SH metals (b) and 2D electron states with spin-orbit splitting (c). In SH metals, the spin polarized hot electrons are deflected sideways after they are injected from the FM electrode. For 2D states, the majority and minority electrons tunnel into different sub-bands with different parallel wavevectors. In $\mathbf{b}$, the direction of the electric field is defined by the convention that under a positive bias electrons tunnel into states above the Fermi surface of the NM. d, Schematic of the circuit used in the experiment.

account for this uncertainty, we use a wide range for $\lambda_{\text {sf }}$ in our calculation (1-2.7 nm for Ta (refs 16,17) and $1.5-10 \mathrm{~nm}$ for $\mathrm{Pt}$ (refs 16,18), which reflects the lower and higher bounds of $\lambda_{\mathrm{sf}}$ reported in the recent literature). With those numbers and with the spin polarization calculated from the TMR of standard MTJs, the SH angles are determined to be: $\left|\theta_{\mathrm{SH}}(\mathrm{Ta})\right|=0.05-0.11$ and $\left|\theta_{\mathrm{SH}}(\mathrm{Pt})\right|=0.04-0.09$ (Supplementary Information). Note that as the quality of the tunnelling barrier in the standard MTJs with two $\mathrm{CoFeB}$ electrodes is probably higher compared with that in the NM/oxide/FM junctions, we may have overestimated the spin polarization of our devices. Therefore, the values of the $\mathrm{SH}$ angle reported above should be treated as the lower bound of the actual values of the $\mathrm{SH}$ angle at the Fermi level. The magnitude of the $\mathrm{SH}$ angles has long been a heatedly debated topic in the study of the SHE (ref. 18). Large SH angles that are close to the spin polarization of FM metals usually need to be assumed to explain the magnetic dynamics observed in the $\mathrm{SH}$ spin torque experiments ${ }^{8,11-13}$. Also, electrical transport measurements have reported much smaller values (for example, 0.004 for $\mathrm{Ta}$ and 0.02 for Pt; ref. 16). In this Letter, by employing a pure transport approach, we obtained results that are consistent with previous $\mathrm{SH}$ torque studies, verifying the $\mathrm{SH}$ origins of those experiments. In contrast to previous transport experiments using non-local spin valves ${ }^{16}$, our devices inject spinpolarized current directly into the NM through a tunnel barrier. We thus avoid the complexity of dealing with the loss of the spin current across multiple interfaces and through diffusive metals. Therefore, we believe that $\mathrm{SH}$ tunnelling spectroscopy provides a simple and accurate measurement technique for determining SH angles.

The use of tunnelling electrons has another important advantage-it enables us to apply a finite d.c. bias across the junction and measure the voltage dependence of the ISHE signal. Under finite bias, if the spin relaxation length $\left(\lambda_{\mathrm{sf}}\right)$ is much smaller than the hot-electron attenuation length $\left(\lambda_{\mathrm{at}}\right)$, the measured ISHE signal would mainly come from energetic electrons. In the other extreme, if $\lambda_{\mathrm{sf}} \gg \lambda_{\mathrm{at}}$, the energetic electrons would relax back to the Fermi level before they contribute to the SHE, and thus we would expect to see very little signal variation to reflect the energy dependence of the SHE. When the two length scales are comparable $\left(\lambda_{\mathrm{sf}} \sim \lambda_{\mathrm{at}}\right)$, the observed tunnelling spectra would consist of a superposition of the SHE from both energetic and equilibrium electrons, which would retain the energy dependence of the SHE, albeit with energy-broadened spectral features. According to previous ballistic electron emission experiments, $\lambda_{\mathrm{at}}$ is usually between a few to a few tens of nm for tunnelling electrons in 


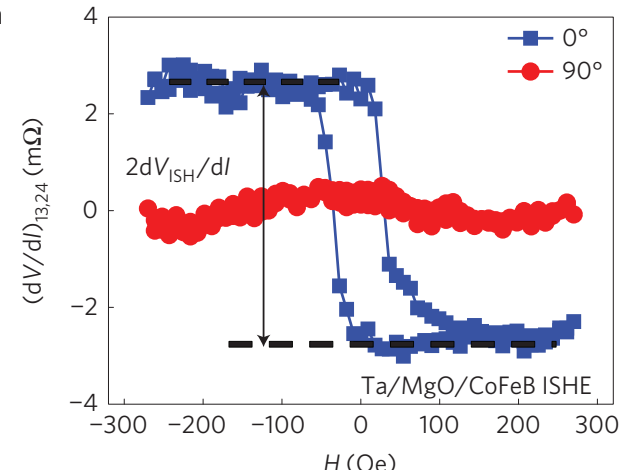

C

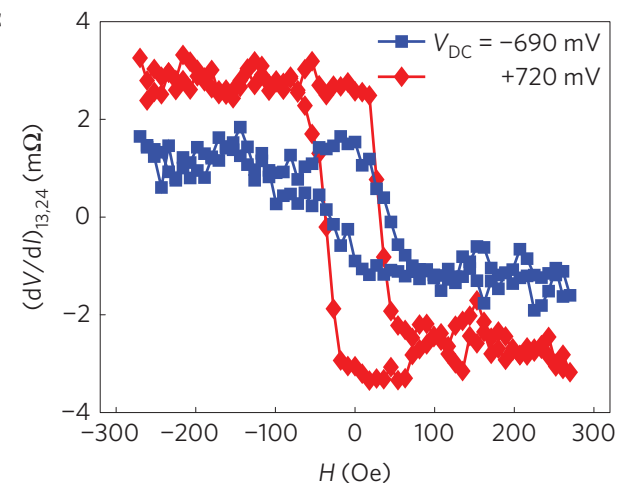

b

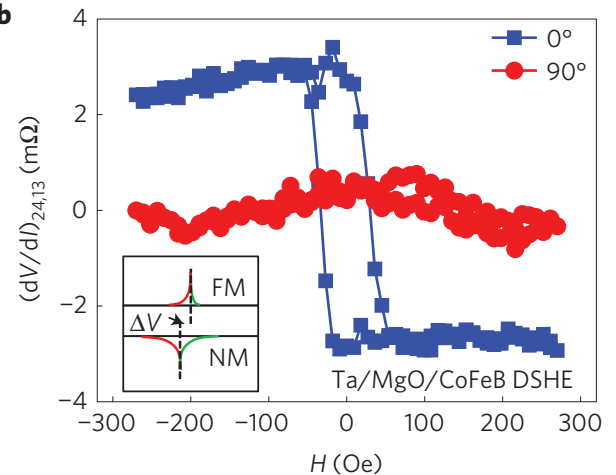

d

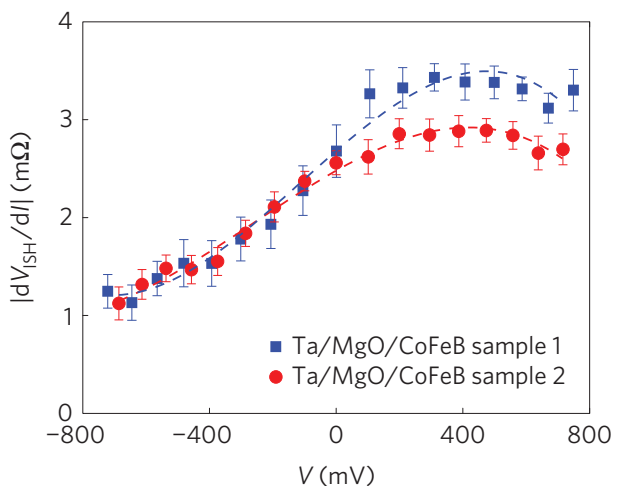

Figure 2 | SHE signal from the Ta/MgO/CoFeB sample. a,b, Transverse resistance $\mathrm{dV} / \mathrm{d} /$ measured in the ISHE (a) and DSHE (b) configurations. The fields are applied along the $x\left(0^{\circ}\right)$ and $y\left(90^{\circ}\right)$ directions in each case. The junction area is $\sim 3 \times 4 \mu \mathrm{m}^{2}$ and the resistance-area product is $\sim 100 \mathrm{k} \Omega \mu \mathrm{m}^{2}$. The differential ISH resistance $d V_{I S H} / d l$ is defined as one half of the field-dependent signal. Inset of $\mathbf{b}$, schematic illustration of the spin accumulation induced by the DSHE at the NM/oxide interface. The red and green curves represent the spin-dependent chemical potentials for the majority and minority spins, respectively. c, ISH resistance $(\mathrm{dV} / \mathrm{d} /)_{13,24}$ measured at two different d.c. biases when the field is swept along $0^{\circ}$. $\mathbf{d}$, The differential ISH resistance $\mathrm{d} V_{\text {ISH }} / \mathrm{d} I$ as a function of the applied d.c. voltage. Two similar devices are shown. The dashed lines represent Taylor polynomial fits for the two samples. The error bars represent the standard deviation of results from ten scans.
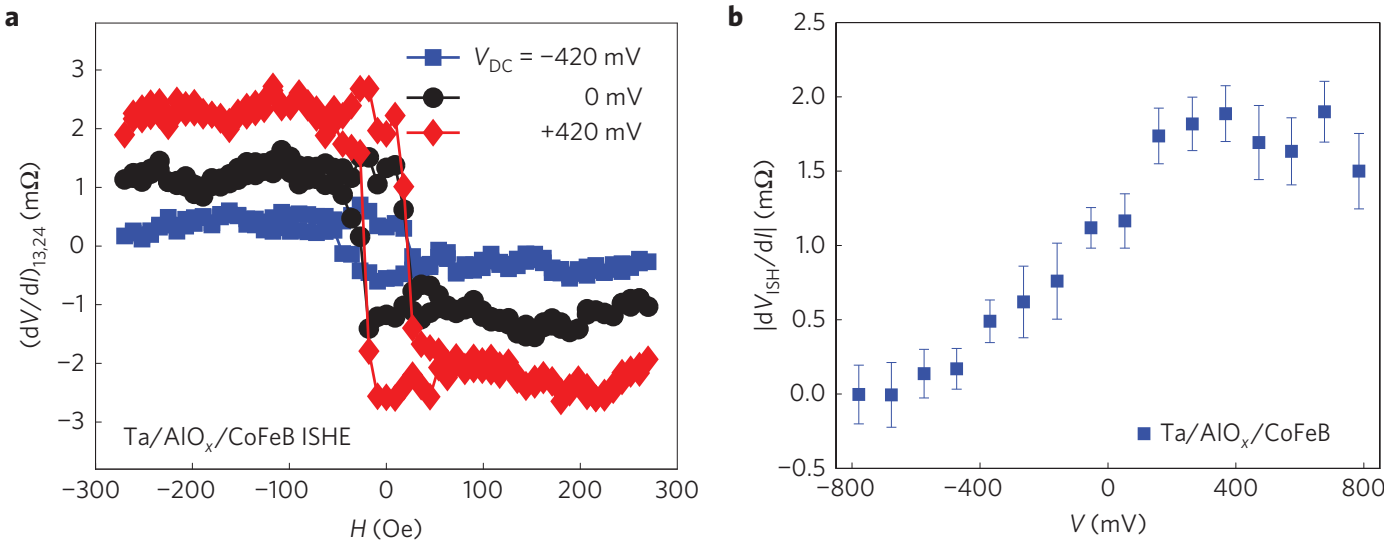

Figure 3 | SHE signal from the $\mathrm{Ta} / \mathrm{AlO}_{x} / \mathrm{CoFeB}$ sample. a, ISH resistance $(\mathrm{dV} / \mathrm{d} /)_{13,24}$ measured at $V_{\mathrm{DC}}=-420 \mathrm{mV}, 0 \mathrm{mV}$ and $+420 \mathrm{mV}$, with the field swept along the $x$ direction $\left(0^{\circ}\right)$. The junction area is $\sim 4 \times 4 \mu \mathrm{m}^{2}$ and the resistance-area product is $\sim 20 \mathrm{k} \Omega \mu \mathrm{m}^{2}$. b, Differential ISH resistance $\mathrm{d} V_{\text {ISH }} / \mathrm{d} /$ as a function of the applied d.c. voltage. The error bars represent the standard deviation of results from ten scans.

transition metals ${ }^{19,20}$. Meanwhile, $\lambda_{\mathrm{sf}}$ is around $1-10 \mathrm{~nm}$ for heavy metals such as $\mathrm{Pt}$ and Ta (refs 17,18,21). Therefore, the two quantities are at least comparable to each other in our studies and the measured signal should contain energy-dependent information from the SHE. Figure $2 \mathrm{c}$ shows the differential ISH resistance $\mathrm{d} V_{\text {ISH }} / \mathrm{d} I$ of $\mathrm{Ta} / \mathrm{MgO} / \mathrm{CoFeB}$ under different d.c. biases. The voltage dependence of $\mathrm{d} V_{\text {ISH }} / \mathrm{d} I$ over the range $\pm 700 \mathrm{mV}$ is summarized in Fig. 2d. We can see that $\mathrm{d} V_{\text {ISH }} / \mathrm{d} I$ decays rapidly under negative bias, but increases under positive bias until the signal saturates around $400 \mathrm{mV}$. On the other hand, the $\mathrm{Pt} / \mathrm{MgO} / \mathrm{CoFeB}$ sample demonstrates a much more symmetric bias-voltage dependence (Fig. 4c,d). We have also measured devices made of W (Fig. 4e) and Ir (Fig. 4f) thin films. At zero d.c. bias, the sign and magnitude of the $\mathrm{SH}$ angles of these two materials (Supplementary Information) agree with previous experiments and calculations ${ }^{10,22}$. At finite bias, the $\mathrm{W}$ samples show a distinct bias asymmetry compared with $\mathrm{Ta}$ and $\mathrm{Pt}$, and the spectral peak is located near the Fermi level, whereas the Ir samples show a monotonic increase in $\mathrm{d} V_{\text {ISH }} / \mathrm{d} I$ with 

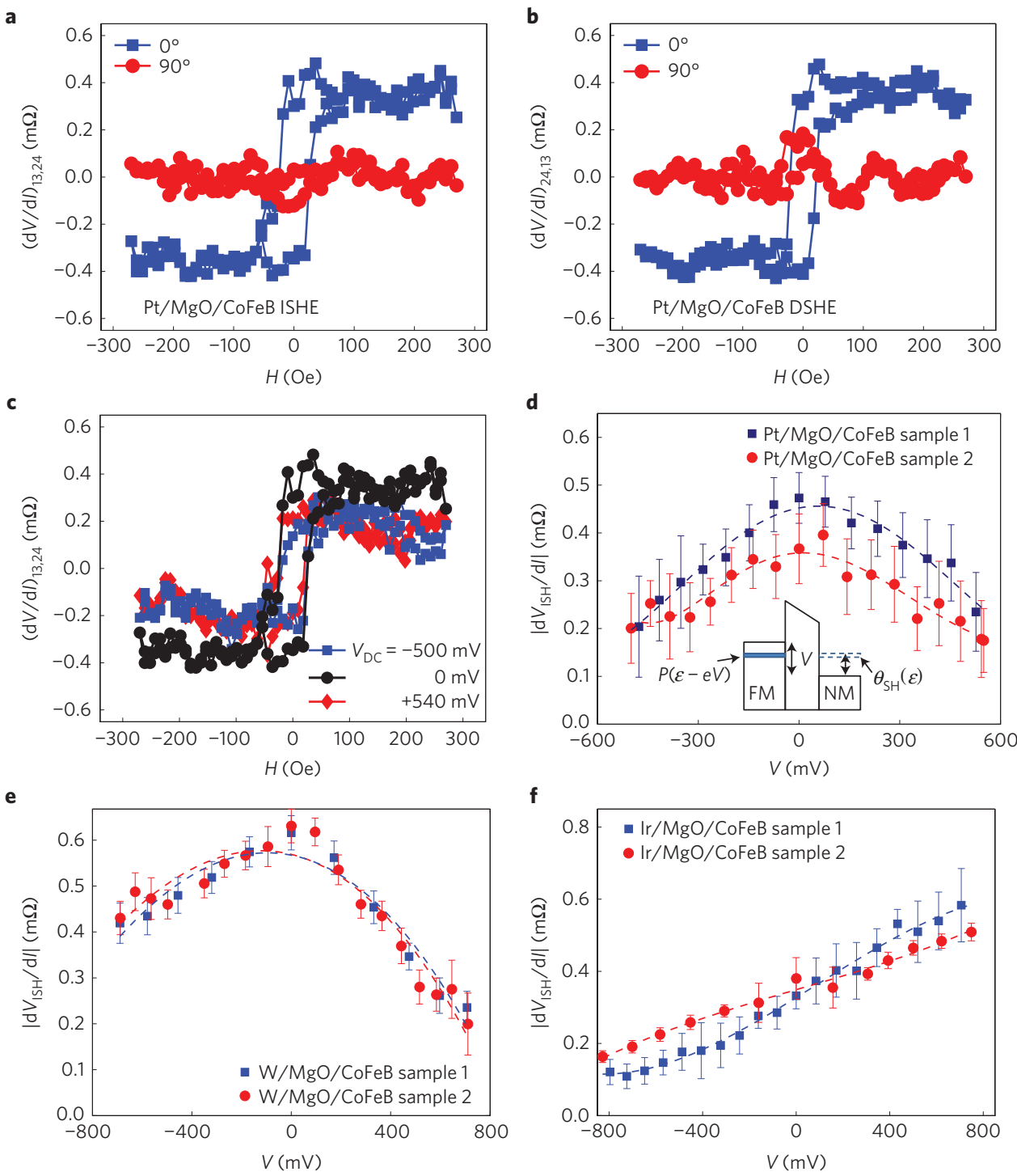

Figure 4 | SHE signal from Pt/MgO/CoFeB sample and voltage dependence of ISHE resistance from $\mathbf{W}$ and Ir samples. a,b, Transverse resistance dV/dl measured in the ISHE (a) and DSHE (b) configurations for the Pt sample. The device has dimensions of $\sim 4 \times 5 \mu^{2}$ and the resistance-area product is $\sim 200 \mathrm{k} \Omega \mu \mathrm{m}^{2}$. In $90^{\circ}$ scans, the small residual resistances at low field in the $\mathrm{dV} / \mathrm{d} /$ versus $\mathrm{H}$ curves are due to the domains aligned along the easy axis $\left(0^{\circ}\right)$ that are not fully compensated, reflecting the multi-domain nature of the CoFeB electrode. $\mathbf{c}$, ISH resistance $(\mathrm{dV} / \mathrm{d} /)_{13,24}$ of the Pt sample measured at $V_{D C}=-500 \mathrm{mV}, 0 \mathrm{mV}$ and $+540 \mathrm{mV}$. d-f. Absolute value of the differential ISH resistance $\mathrm{d} V_{\mathrm{ISH}} / \mathrm{d} /$ as a function of the applied d.c. voltage in Pt (d), $W(\mathbf{e})$ and $\operatorname{Ir}(\mathbf{f})$ samples. The error bars represent the standard deviation of results from ten scans. Two devices are shown for each material. Inset of $\mathbf{d}$, schematic illustration of the tunnel junction under a d.c. bias voltage of $V . P(\varepsilon-e V)$ and $\theta_{S H}(\varepsilon)$ represent the spin polarization and the spin Hall angle for electrons with the corresponding energy, respectively.

bias voltage within the voltage range of the experiment. We note that, compared with $\mathrm{Ta} / \mathrm{MgO} / \mathrm{CoFeB}$, the $\mathrm{Ta} / \mathrm{AlO}_{x} / \mathrm{CoFeB}$ sample shows a very similar bias asymmetry in $\mathrm{d} V_{\text {ISH }} / \mathrm{d} I$ (Fig. 3a,b). The faster drop in the $\mathrm{d} V_{\text {ISH }} / \mathrm{d} I(V)$ value under negative bias in the $\mathrm{AlO}_{x}$ sample is consistent with a stronger voltage dependence of spin polarization across the $\mathrm{AlO}_{x}$ tunnel barriers (Supplementary Fig. 2c,d). The fact that devices made from amorphous $\mathrm{AlO}_{x}$ barriers and coherent $\mathrm{MgO}$ barriers show similar spectral features also indicates that the major contribution to the voltage dependence of $\mathrm{d} V_{\text {ISH }} / \mathrm{d} I(V)$ may not come from the effect of tunnelling matrix elements.

Under finite bias, the DOS and tunnelling probability of tunnelling electrons may both change with the applied voltage. Correspondingly, the tunnelling current and the induced transverse voltage would also vary. In our experiment, however, the nonlinearity of the $I-V$ characteristics has been taken into account by normalizing the ISH voltage against the applied a.c. current. Therefore, the voltage dependence of current does not contribute to the voltage dependence of $\mathrm{d} V_{\text {ISH }} / \mathrm{d} I$. Another factor that can contribute to the voltage dependence is the spin polarization $P(V)$. In our experiment, $P(V)$ alone cannot explain the drastically different bias asymmetries observed in devices made using different $5 d$ transition metals, which have nominally the same barrier and FM electrode. Moreover, the voltage-dependent spin polarization in standard MTJs can be estimated using their TMR value, which provides a guideline for estimating $P(V)$ of the NM/oxide/FM junctions. It can be shown that the variation of $P$ is relatively small ( $\leq 30 \%$; Supplementary Information), not enough to account for the comparatively large changes in the ISH signal $(\geq 50 \%$ in $\mathrm{Pt}$, even larger in $\mathrm{Ta}$, Ir, and $\mathrm{W}$ ). Although the above discussions strongly indicate that the SHE is not fully masked by the energy dependence of the other factors, a quantitative determination of the 
energy-dependent SH angle requires more experimental data on the voltage dependence of the spin polarization and the spin/energy relaxation length, as well as a rigorous treatment which takes into account the band structure of both electrodes and the tunnelling matrix of the barrier.

A comparison can be made between the measured voltagedependent SHE signal and theoretical calculations based on the band structures of the materials. For Pt, results in Fig. $4 \mathrm{~d}$ qualitatively agree with previous calculations $s^{9,10}$ in which the intrinsic SH conductivity was shown to peak close to the Fermi surface. This is consistent with the fact that, for the intrinsic SHE in $\mathrm{Pt}$, the dominant contribution comes from the avoided crossings of the nearly degenerate bands located near the Fermi level, where the uncompensated Berry curvature gives rise to a large SH conductivity9. For other metals, further firstprinciples calculations using the same crystalline structures as those of our films (for example, the $\beta$ phase for Ta) are necessary to explain the voltage dependence found in the tunnelling spectra.

Besides the SHE, which is generally calculated using the bulk electronic structure of the corresponding material, spin-orbit interactions can also induce splitting in localized surface states, as observed in many heavy-element metals ${ }^{23-25}$ by means of angle resolved photoemission spectroscopy (ARPES). A similar transverse voltage $^{26}$ can be generated as a result of spin-momentum locking (Fig. 1c). Our energy-dependent $\mathrm{d} V_{\text {ISH }} / \mathrm{d} I$ measurement provides a method to distinguish between those two mechanisms. For example, in $\mathrm{Pt}$, previous calculations and experiments show that the bottom of surface bands is located $\sim 300 \mathrm{meV}$ above the Fermi surface ${ }^{25,27}$. Within the resolution of our experiment, we observe no sharp transition or change of slope around that energy level. Moreover, we can compare the $\mathrm{d} V_{\text {ISH }} / \mathrm{d} I(V)$ tunnelling spectra of W (Fig. 4e) and Ir (Fig. 4f) with the ARPES spectra, in which the spinorbit-interaction-induced surface band splitting has been observed earlier ${ }^{23,24}$. That the spectral peaks in $\mathrm{d} V_{\text {ISH }} / \mathrm{d} I(V)$ do not agree with the location of the surface bands observed by ARPES in these metals indicates that the tunnelling transverse signal should be mainly attributed to the SHE in the projected bulk bands. Moreover, the consistency between the SH angles measured in our experiment and those in NM/FM bilayer spin-orbit torque measurements also implies that the anti-damping torque observed in those experiments shares the same bulk band origin.

The SHE spectra provide guidelines for further improving the magnitude of the SHE by means of electronic structure engineering, for example, by adjusting the Fermi surface position through alloying or chemical compound formation. Because of the impedance matching capability of the tunnel junctions, besides metals, the SHE tunnelling spectroscopy can also be used to investigate semiconductors. It will be particularly useful for probing spin-orbit interactions in topological insulators or two-dimensional (2D) electron gases where surface states dominate. In the former case, the capability of applying finite biases will enable the detection of electrons close to the Dirac point.

\section{Methods}

Sample fabrication. The full stacks of substrate/SH metal (7)/oxide tunnel barrier (1.5-2.5)/ $\mathrm{Co}_{20} \mathrm{Fe}_{60} \mathrm{~B}_{20}$ (4)/ $\mathrm{Ta}$ (5)/Ru (5) (units in nm) were first deposited by sputtering and then patterned into the shape shown in Fig. 1a by a combination of photolithography and ion beam etching. The etching stops slightly below the $\mathrm{SH}$ metal/oxide interface. The $\mathrm{CoFeB}$ layer has in-plane anisotropy because of the chosen thickness. Post-deposition annealing was used to optimize the tunnelling spin polarization. Top contacts made from a layer of $\mathrm{Ta}(5 \mathrm{~nm}) / \mathrm{Cu}(90 \mathrm{~nm}) / \mathrm{Pt}(20 \mathrm{~nm})$ were deposited after the side of the tunnel junction was encapsulated with $\mathrm{SiO}_{2}$. The resistance-area (RA) product of tunnel barriers in our samples ranges between $20 \mathrm{k} \Omega \mu \mathrm{m}^{2}$ and $300 \mathrm{k} \Omega \mu \mathrm{m}^{2}$. The tunnel junctions have rectangular shapes with their easy axis aligned along the $x$ direction. The lateral dimensions of tunnel junctions vary between 3 and 8 um and the channel width of the SH metal is $8 \mu \mathrm{m}$.
Circuit and measurement scheme. In the circuit, we used an audio transformer to inject d.c. and a.c. currents simultaneously into the tunnel junction. The a.c. current is provided by the internal oscillator of the lock-in amplifier. The frequency of the a.c. current $I_{\mathrm{AC}}$ was set to be around $1 \mathrm{kHz}$. The amplitude of $I_{\mathrm{AC}}$ was read out by measuring the voltage of the serial sensing resistor $R_{\text {sense }}=10 \Omega$ and was maintained around $10 \mu \mathrm{A}$ in our experiments. For our $8 \mathrm{k} \Omega$ devices, this corresponds to a $\sim 80 \mathrm{mV}$ a.c. voltage across the junction at zero d.c. bias, which determines the energy resolution of our technique. To avoid electrical breakdown of the tunnel junction, another serial resistor $R_{\text {limit }}$ was used to limit the a.c. voltage distribution on the device. To measure the transverse a.c. voltage, we first amplified the signal between leads 2 and 4 through a pre-amp, so that the floated voltage is converted into a signal relative to the ground. Then we connected the output of the pre-amp to one end of the input channel of a lock-in amplifier and the resistor bridge to the other end of the lock-in input to compensate for parasitic signals due to misalignment. The absolute value of the parasitic resistance is $<20 \Omega$ for Ta and $<4 \Omega$ for $\mathrm{Pt}, \mathrm{W}$ and Ir. Both positive and negative values have been observed for devices on different chips, indicating randomness in the pattern misalignment. We measured the ISH voltage as a function of the applied sensing current $I_{\mathrm{AC}}$ within a wide range and a good linear dependence was observed. This excludes the possibility that the observed signal comes from thermal effects (Supplementary Information). For energy dependence measurements, we use a d.c. current source to provide the bias voltage. The d.c. voltage of the device was measured with a voltmeter connected in parallel with the output of the current source. As the resistance of $R_{\text {sense }}$ is much smaller than that of the device, the measured voltage is approximately the same as the voltage drop across the junction. All the measurements were done at room temperature.

Received 24 January 2014; accepted 22 May 2014; published online 20 July 2014

\section{References}

1. Dyakonov, M. I. \& Perel, V. I. Current-induced spin orientation of electrons in semiconductors. Phys. Lett. A 35, 459-460 (1971)

2. Hirsch, J. E. Spin Hall effect. Phys. Rev. Lett. 83, 1834-1837 (1999).

3. Zhang, S. F. Spin Hall effect in the presence of spin diffusion. Phys. Rev. Lett. 85, 393-396 (2000).

4. Valenzuela, S. O. \& Tinkham, M. Direct electronic measurement of the spin Hall effect. Nature 442, 176-179 (2006).

5. Kimura, T., Otani, Y., Sato, T., Takahashi, S. \& Maekawa, S. Room-temperature reversible spin Hall effect. Phys. Rev. Lett. 98, 156601 (2007).

6. Kajiwara, Y. et al. Transmission of electrical signals by spin-wave interconversion in a magnetic insulator. Nature 464, 262-266 (2010).

7. Miron, I. M. et al. Perpendicular switching of a single ferromagnetic layer induced by in-plane current injection. Nature 476, 189-193 (2011).

8. Liu, L. Q. et al. Spin torque switching with the giant spin Hall effect of tantalum. Science 336, 555-558 (2012).

9. Guo, G. Y., Murakami, S., Chen, T. W. \& Nagaosa, N. Intrinsic spin Hall effect in platinum: First-principles calculations. Phys. Rev. Lett. 100, 096401 (2008).

10. Tanaka, T. et al. Intrinsic spin Hall effect and orbital Hall effect in $4 \mathrm{~d}$ and $5 \mathrm{~d}$ transition metals. Phys. Rev. B 77, 165117 (2008).

11. Liu, L., Lee, O. J., Gudmundsen, T. J., Ralph, D. C. \& Buhrman, R. A. Current-induced switching of perpendicularly magnetized magnetic layers using spin torque from the spin Hall effect. Phys. Rev. Lett. 109, 096602 (2012).

12. Emori, S., Bauer, U., Ahn, S. M., Martinez, E. \& Beach, G. S. Current-driven dynamics of chiral ferromagnetic domain walls. Nature Mater. 12, 611-616 (2013).

13. Fan, X. et al. Observation of the nonlocal spin-orbital effective field. Nature Commun. 4, 1799 (2013).

14. Wolf, E. L. Principles of Electron Tunneling Spectroscopy (Oxford Univ. Press, 2012).

15. Takahashi, S. \& Maekawa, S. Spin injection and detection in magnetic nanostructures. Phys. Rev. B 67, 052409 (2003).

16. Morota, M. et al. Indication of intrinsic spin Hall effect in $4 \mathrm{~d}$ and $5 \mathrm{~d}$ transition metals. Phys. Rev. B 83, 174405 (2011)

17. Boone, C. T., Nembach, H. T., Shaw, J. M. \& Silva, T. J. Spin transport parameters in metallic multilayers determined by ferromagnetic resonance measurements of spin-pumping. J. Appl. Phys. 113, 153906 (2013).

18. Liu, L., Buhrman, R. \& Ralph, D. Review and analysis of measurements of the spin Hall effect in platinum. Preprint at http://arxiv.org/abs/1111.3702 (2011).

19. Niedermann, P., Quattropani, L., Solt, K., Maggio-Aprile, I. \& Fischer, O. Hot-carrier scattering in a metal: A ballistic-electron-emission microscopy investigation on PtSi. Phys. Rev. B 48, 8833-8839 (1993). 
20. Weilmeier, M. K., Rippard, W. H. \& Buhrman, R. A. Ballistic electron transport through $\mathrm{Au}(111) / \mathrm{Si}(111)$ and $\mathrm{Au}(111) / \mathrm{Si}(100)$ interfaces. Phys. Rev. B 59, R2521-R2524 (1999).

21. Zhang, W. et al. Determination of the Pt spin diffusion length by spin-pumping and spin Hall effect. Appl. Phys. Lett. 103, 242414 (2013).

22. Pai, C-F. et al. Spin transfer torque devices utilizing the giant spin Hall effect of tungsten. Appl. Phys. Lett. 101, 122404 (2012)

23. Hochstrasser, M., Tobin, J. G., Rotenberg, E. \& Kevan, S. D. Spin-resolved photoemission of surface states of W(110)- $(1 \times 1)$ H. Phys. Rev. Lett. 89, 216802 (2002)

24. Varykhalov, A. et al. $\operatorname{Ir}(111)$ surface state with giant Rashba splitting persists under graphene in air. Phys. Rev. Lett. 108, 066804 (2012).

25. Bendounan, A. et al. Evolution of the Rashba spin-orbit-split Shockley state on Ag/Pt(111). Phys. Rev. B 83, 195427 (2011).

26. Sánchez, J. C. R. et al. Spin-to-charge conversion using Rashba coupling at the interface between non-magnetic materials. Nature Commun. 4, 2944 (2013).

27. Wiebe, J. et al. Unoccupied surface state on $\mathrm{Pt}(111)$ revealed by scanning tunneling spectroscopy. Phys. Rev. B 72, 193406 (2005).

\section{Acknowledgements}

The authors thank D. C. Worledge, G. Hu and I. Garate for helpful discussions and assistance in manuscript writing.

\section{Author contributions}

L.L. deposited the films and fabricated the devices. L.L. and C-T.C. carried out the electrical measurements. L.L. carried out data analysis and modelling with the help and guidance of J.Z.S. L.L. and C-T.C. wrote the manuscript. All authors discussed the results and commented on the manuscript.

\section{Additional information}

Supplementary information is available in the online version of the paper. Reprints and permissions information is available online at www.nature.com/reprints.

Correspondence and requests for materials should be addressed to L.L.

\section{Competing financial interests}

The authors declare no competing financial interests. 